\title{
Refractive index sensor based on slot waveguide cavity
}

\author{
A. Cosentino \\ armando.cosentino@epfl.ch \\ Q. Tan
}

M. Roussey

\section{H. P. Herzig}

\author{
Optics \& Photonics Technology Laboratory, Ecole Polytechnique Fédérale de Lausanne (EPFL), \\ 2000 Neuchâtel, Switzerland \\ Optics \& Photonics Technology Laboratory, Ecole Polytechnique Fédérale de Lausanne (EPFL), \\ 2000 Neuchâtel, Switzerland \\ Department of Physics and Mathematics, University of Eastern Finland, 80100 Joensuu, Finland
}

The experimental study of a gold slot waveguide cavity is presented. The resonance of this cavity working in the telecom wavelength range is highly dependent on the refractive index of the medium located in or around the slots array, because of the high confinement of the electromagnetic field in the structure. We will demonstrate the application of this structure to local refractive index sensors at the nanoscale. The measured sensitivity of this device is $S=730 \mathrm{~nm} / \mathrm{RIU}$ (refractive index unit). The structure has been optimized by adding another array of slots cascaded with the first one. The consequence is an improvement in the time efficiency of the experiments. A discussion about the effect of the volume of liquids used and the filling percentage of the slots by the liquids is also presented as parameters affecting the measurements and the sensitivity of the sensor.

[DOI: http://dx.doi.org/10.2971/jeos.2012.12039]

Keywords: Nanostructure, sensing, characterization, waveguide

\section{INTRODUCTION}

In optical sensing, refractive index (RI) detection [1] is a straightforward method used in various applications [2], for which a variety of structures has been studied [3]-[7]. Following recent advancements in technology, the development of miniaturized devices is becoming increasingly prevalent for the exploration of compact and complex systems. Considering the miniaturization, subwavelength nanostructures allow the detection of highly localized RI variations. Photonic crystal [8] and plasmonic [9] based sensors are examples of localized sensors. Nanostructured metallic devices exhibit the ability to confine and enhance the electromagnetic field in volumes smaller than the diffraction limit. The field localization enhances the light-analyte interaction in the case where we want to probe very small volumes. This is useful to investigate localized biomolecular interactions in medicine and pharmaceutical research. Besides miniaturization, a planar sensor like the one herein presented is integrable with different optical parts and fluidic components and allows multiplexing.

\section{PRINCIPLE AND EXPERIMENT}

\subsection{Design}

The proposed structure is a waveguide-based sensor combined with a periodic metallic slot array nanocavity. Previous studies of this variety of nanostructures were done by Dionne et al. [10] and Veronis and Fan [11] who provided a new in- sight on subwavelength slots and generation of plasmonic guidance in a few tens of nanometers in metal-insulator-metal (MIM) cavities [12]. In a previous work [13], we have shown theoretically and experimentally how the geometrical parameters of this structure affect the behavior of light in the cavity. Moreover we have proved theoretically the potential application to refractive index sensing of this device. It is indeed well known that a variation of the refractive index of the medium surrounding and inside the cavity changes the position of its resonance. The simplicity of the chosen structure permits the study of the effect of the different parameters (geometrical or environmental) and to tune the resonance easily. In this paper, we propose the experimental measurement of the calculated sensitivity (726 nm/RIU (refractive index unit)) [13] obtained by Finite Difference Time Domain Calculation [14]. In addition, we discuss the effect of the infiltration of the liquid inside the slots and the role played by the amount of analyte used. An optimized structure which is composed of two cascaded arrays of slots allows a higher contrast.

The device is presented in Figure 1(a). It is composed of a classic dielectric ridge waveguide $\left(\mathrm{SiO}_{2} / \mathrm{Si} / \mathrm{SiO}_{2}\right)$ on top of which an array of slots has been fabricated in a $20 \mathrm{~nm}$-thick gold layer. The cavity is called Slot Waveguide Cavity (SWC) and its geometrical parameters are a period $p=500 \mathrm{~nm}$, a slot width $w=30 \mathrm{~nm}$, and a length $l=700 \mathrm{~nm}$.

To fabricate the device, a wet oxided Si wafer is coated with 


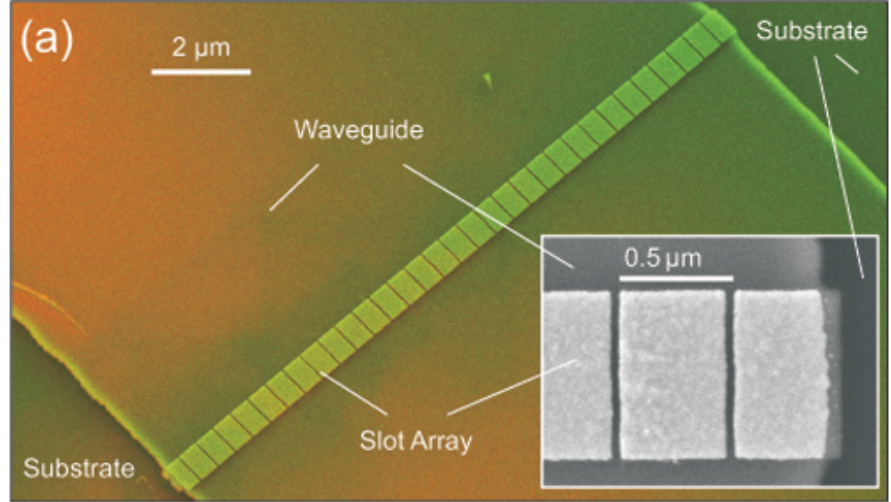

(b)

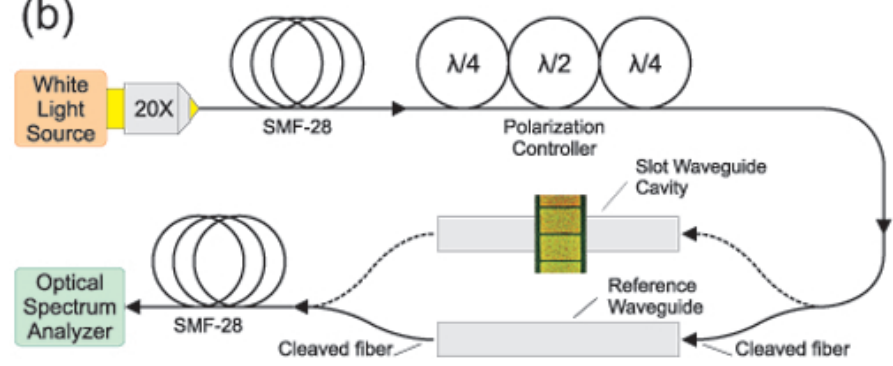

FIC. 1 (a) SEM image of the SWC structure using the mixing of the SEM signals from the upper (SEI detector - red) and the lower (LEI detector - green) secondary electron image sensors. The right inset shows the details of the SWC; (b) Scheme of the experimental setup for the characterization of the transmission through the SWC.

amorphous Si (by Low Pressure Chemical Vapor Deposition, LPCVD) and $\mathrm{SiO}_{2}$ (using sputtering). E-beam lithography, gold deposition and lift-off method [15] are employed to draw the cavity. The wafer is spun with the e-beam resist ZEP500A (Zeon Co.) and the pattern is exposed using a Vistec EBPG5000 e-beam pattern generator at the acceleration voltage of $100 \mathrm{kV}$. The gold layer is deposited by means of e-beam evaporation. The resist and the gold excess are sonicated and washed away in an acetone bath. The ridge waveguide is fabricated by means of photolithography and reactive ion etching (RIE).

\subsection{Experimental setup}

The scheme of the experimental setup used for the characterization of the device is presented in Figure 1(b). The light is coupled to the cavity thanks to the evanescent wave coming from the dielectric waveguide. The $\mathrm{SiO}_{2}$ layer above the waveguide acts as a spacer, which allows us to optimize the coupling. Note that only TM polarized light (magnetic field perpendicular to the plane of the slots) is affected by the cavity. The response of the structure is a resonant dip which is observed in the transmission spectrum at the output of the waveguide. In the rest of the paper we will denote as $\lambda_{d}$ the spectral position of this resonance.

Light coming from a supercontinuum source (Koheras SuperK Extreme) is injected into the ridge dielectric waveguide and then the light is collected at the output and sent to an Optical Spectrum Analyzer (Ando AQ-6315B). This technique allows us to obtain the spectral response of the structure from $\lambda=1200 \mathrm{~nm}$ to $\lambda=1700 \mathrm{~nm}$ in one single measurement. Each transmission spectrum through the cavity has been nor-
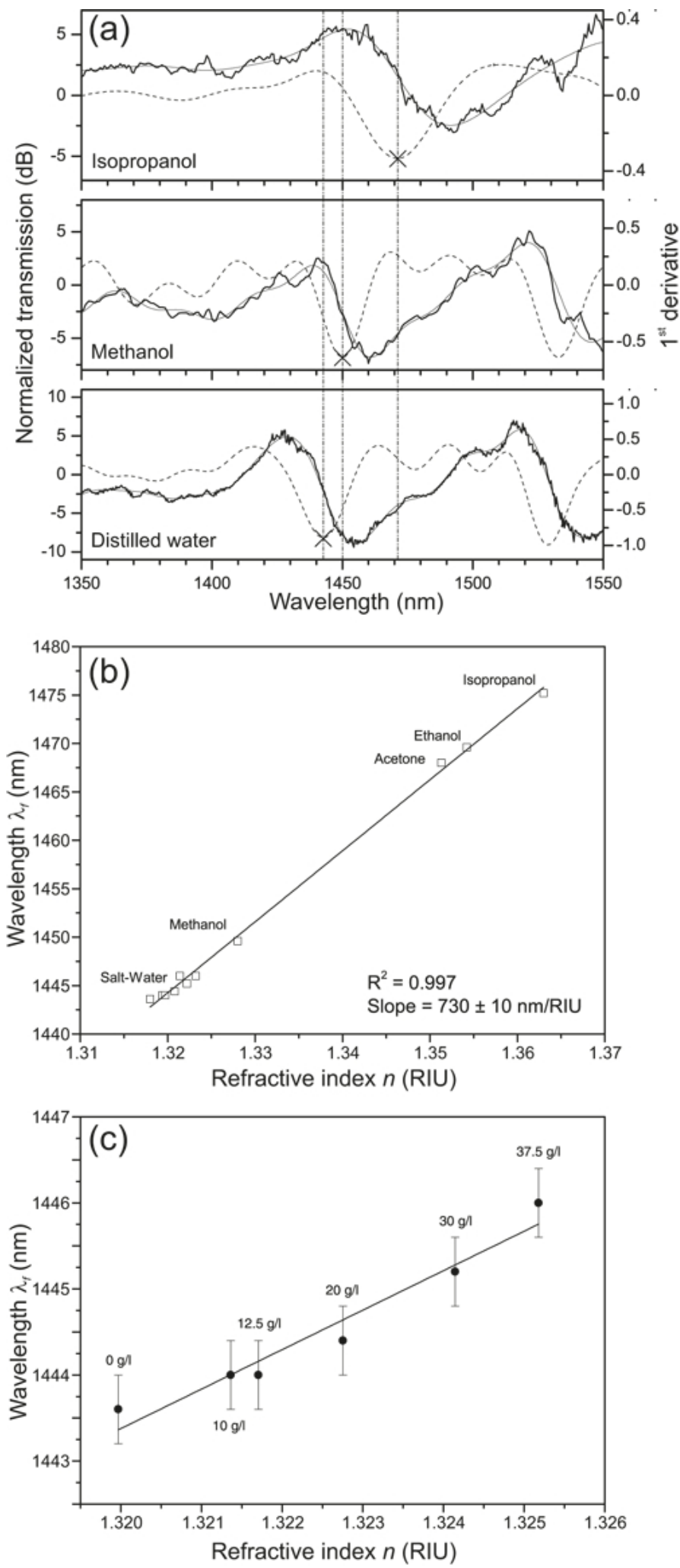

FIG. 2 (a) Experimental transmission spectra related to isopropanol, methanol and distilled water. Solid thick lines are the measured spectra, thin solid lines are the corresponding filtered curves, and dashed lines corresponds to the first derivatives. (b) Calibration curve of the sensor made by measuring the position of the resonance for 11 analytes. (c) Zoom-in on the data corresponding to the salt-water solution.

malized by the spectrum through a waveguide without a cavity on top. We can then avoid a part of the background light, the spectral fluctuation of the white light source and the spectral profile of the waveguide. After normalization, only the ef- 
fect of the slot waveguide cavity remains on the transmission spectrum.

\subsection{Measurement of the sensitivity}

The device has been designed to be a refractive index variation sensor for liquids. The aim of this section is to quantify the sensitivity of the structure to the change of refractive index by using different liquids as analyte. For each liquid, a droplet is deposited on the device, then four measurements are recorded and finally the sample is cleaned.

The results are presented in Figure 2. Figure 2(a) illustrates how the shift of the resonance is determined. First the measured spectrum (thick solid line) is filtered using Fast Fourier Transform low-pass filtering (thin solid line). The first derivative (dashed line) is then calculated in order to determine the position $\left(\lambda_{f}\right)$ of the inflection point of the resonance dip. Note that this method is more convenient than simply measuring the position $\lambda_{d}$ corresponding to the minimum of the dip. Indeed, the quality factor of the resonance is low and the minimum can be difficult to determine. This is shown for three analytes: isopropanol $(n=1.363)$, methanol $(n=1.328)$ and distilled water $(n=1.318)$. We can see on these graphs that the dip shifts to the higher wavelengths when the refractive index of the medium filling and surrounding the slots increases.

The measurements have also been done for ethanol $(n=1.3542)$ and acetone $(n=1.3513)$. The characteristic positions $\left(\lambda_{f}\right)$ of the resonances have been reported on the graph in Figure 2(b) in function of the refractive index of each analyte. We obtain then a calibration curve of our device.

In order to increase the number of experimental points corresponding to a smaller variation of the refractive index, we have also performed the measurements using different solutions of salt $(\mathrm{NaCl})$ in water. Indeed, the refractive index of the solution depends on the concentration of salt. The measured data is presented in Figure 2(c) for mass concentration of salt of $0,10.0,12.5,20.0,25.0,35.0$ and $37.5 \mathrm{~g} / \mathrm{L}$.

Considering a linear relation between the shift of the resonance $(\Delta \lambda)$ and the variation of the refractive index $(\Delta n)$, we have measured a sensitivity $S=\Delta \lambda / \Delta n=730 \pm 10 \mathrm{~nm} / \mathrm{RIU}$. This value is in very good agreement with the theory, which predicted a sensitivity of $726 \mathrm{~nm} / \mathrm{RIU}$.

We can finally remark that the response of the sensor is linear over a wide range of refractive index (from $n=1.318$ to $n=1.363$, here). It corresponds to the refractive index of most of the mixtures based on water, which is ideal for biological applications.

\subsection{Cascaded slot waveguide cavity}

In order to optimize the shape of the dip, i.e. to increase its amplitude, we have compared the single slot waveguide to the cascaded cavity. It is composed of two arrays of slots $(w=20 \mathrm{~nm}, h=30 \mathrm{~nm}, l=700 \mathrm{~nm}$, and $p=500 \mathrm{~nm}) \mathrm{sep}-$ arated by a distance of $d=300 \mathrm{~nm}$. In this case, the modes propagating in the first array are coupled to the second and (a)
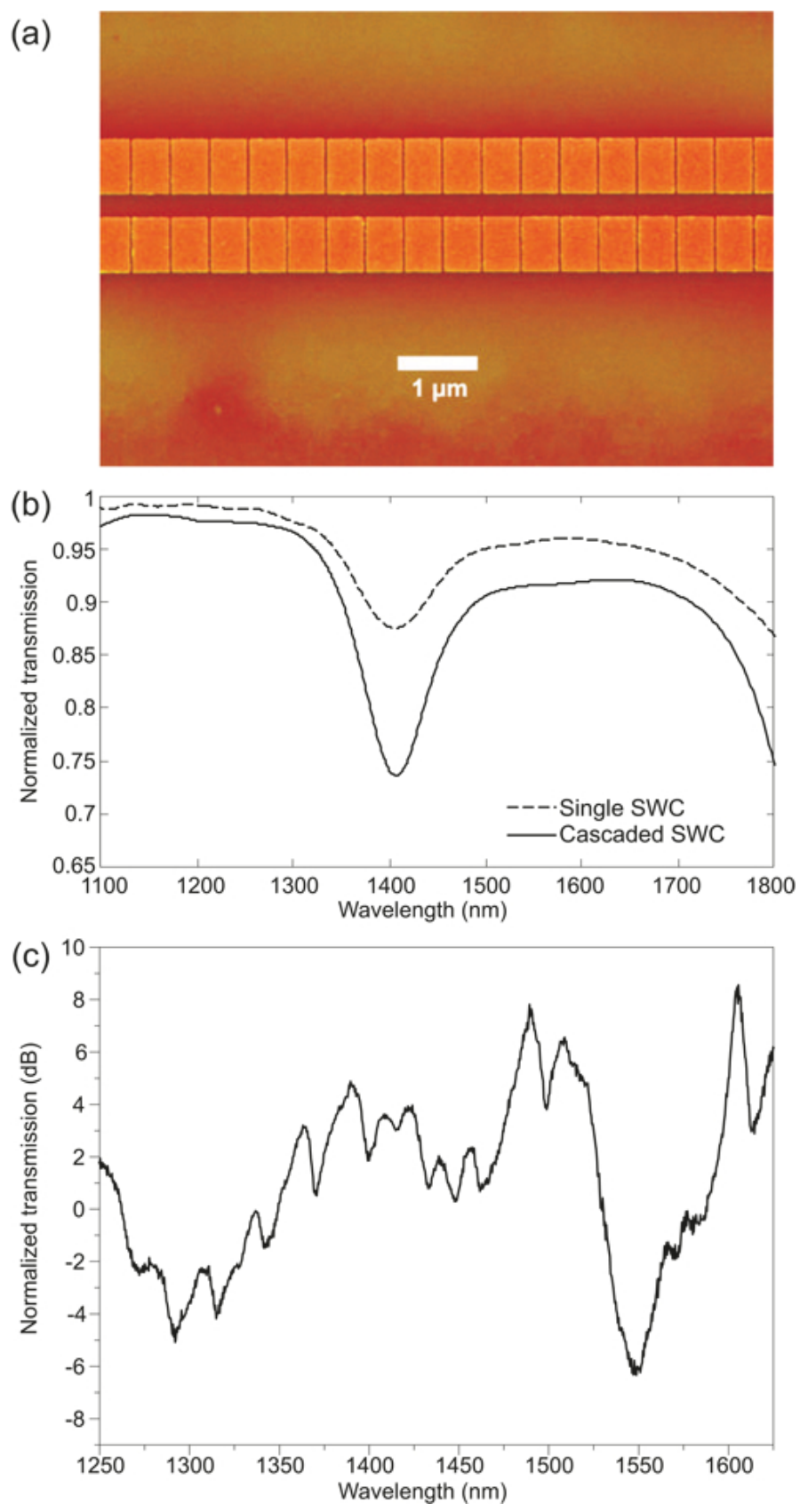

FIC. 3 (a) SEM picture (mixed signals) of the cascaded slot waveguide cavity; (b) Theoretical spectra, calculated by FDTD, of the cascaded SWC compared to the classical single SWC; (c) Measured transmission spectra of the cascaded slot waveguide (SEM picture) when filled with ethanol.

the consequence is an increase of $50 \%$ of the contrast of the dip observed in the transmission spectra.

Figure 3(a) shows the SEM picture of the fabricated structure and Figure 3(b) is the calculated transmission spectra for one and two arrays of slots. Measurements have been performed on the sample with different analytes and the transmission spectrum when ethanol is filling and covering the cavity is presented in Figure 3(c).

We have obtained an increase around $40 \%$ of the amplitude of the dip, which corresponds to theory and makes its observation easier. Nevertheless, the sensitivity of this sensor remains the same as in the case of the single slot array. The improvement of signal-to-noise ratio tends to facilitate the determina- 


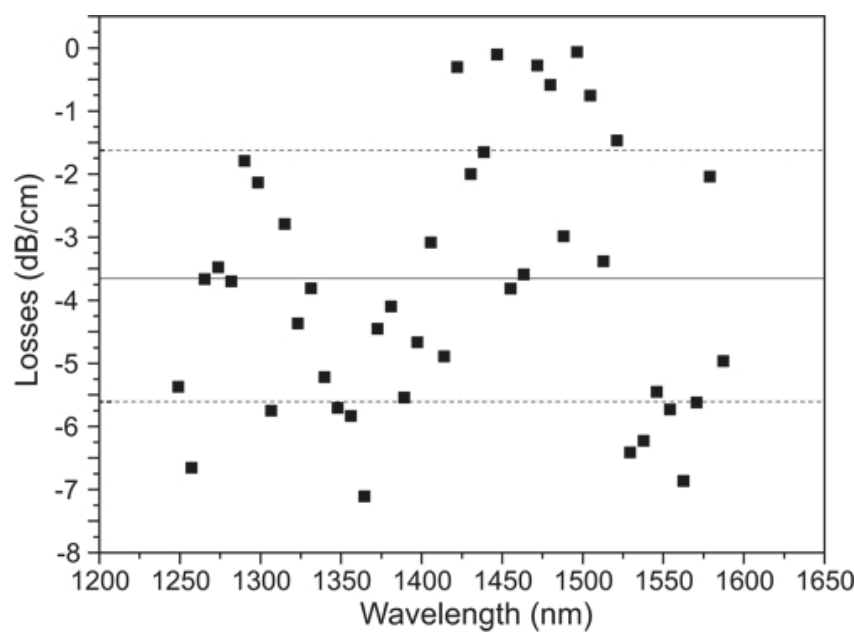

FIG. 4 Measured losses in the waveguides without cavity in function of the wavelength.

tion of the resonance and the time efficiency during the experiments.

\section{DISCUSSION AND RESULTS}

The effect of the geometrical parameters on the resonance position have been detailed in our previous work [13]. It explains mainly the variations between the experimental and the theoretical curves. In addition to the geometry of the cavity itself, some parameters may influence the shape and position of the resonance. We propose to discuss here three of them: the losses in the waveguide, directly linked to the amplitude of the resonance; the size of the droplet of the liquid deposited on the sensor; the percentage of infiltration of the liquids inside the slots.

\subsection{Losses in the waveguide}

In order to characterize the losses in the waveguide, we have measured the transmission through waveguides without cavity and having different lengths $(2.5 \mathrm{~mm}, 3.5 \mathrm{~mm}, 5.5 \mathrm{~mm}$, and $10.5 \mathrm{~mm})$. The results are summarized in Figure 4. It corresponds to an average value of several measurements made on the four different waveguides.

One can see that the average loss value is around $-4 \mathrm{~dB} / \mathrm{cm}$, which is common for this type of waveguide [16]. Nevertheless, the waveguides have been designed to be optimized around the resonance wavelength of the cavity $(\lambda \simeq 1400 \mathrm{~nm})$, for which the losses are reduced. It is obtained by adjusting the thickness of the slab Silicon waveguide $\left(h_{\mathrm{Si}}=220 \mathrm{~nm}\right)$ and the thickness of the cladding $\mathrm{SiO}_{2}$ layer $\left(h_{\mathrm{SiO}_{2}}=80 \mathrm{~nm}\right)$. Note that this last layer plays also the role of a spacer to optimize the coupling of the evanescent wave into the cavity, which means that a compromise had to be found.

\subsection{Effect of the size of the droplet}

A major difference between the tested liquids is their wettability. Indeed, to draw the calibration curve, we have performed the measurements using solvents and salt-water solu-

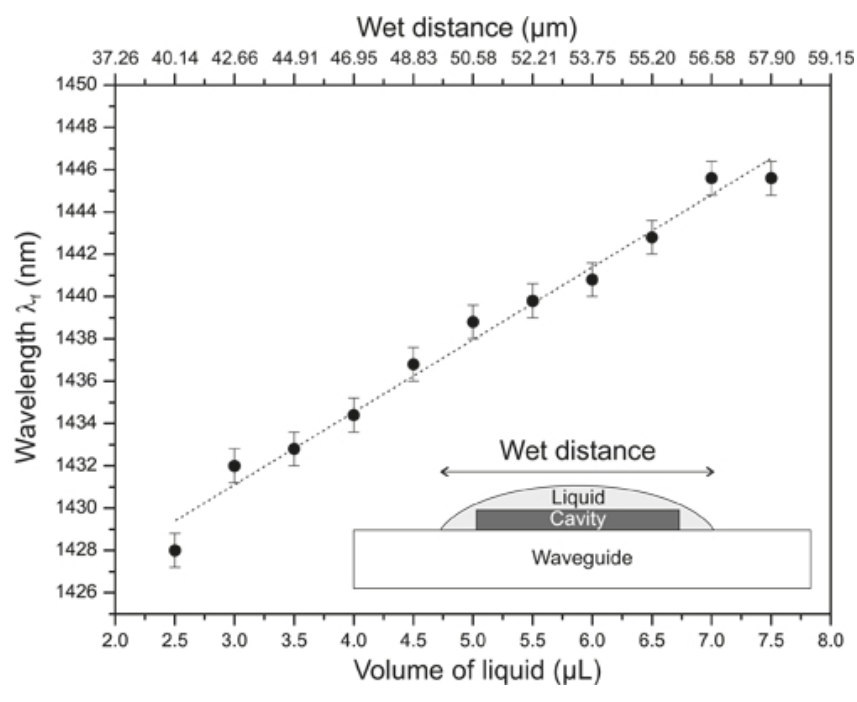

FIC. 5 Influence of the amount of analyte deposited on the sample: measurement of the resonance shift.

tion. While organic solvents completely wet the surface, water forms a well-defined droplet with a contact angle around $\theta=22^{\circ}$ [17]. In order to evaluate the effect of the size of the droplet on the response of the cavity, we have performed a serial of measurements with different volume of water on top of the cavity and observed the variation of the resonance.

Figure 5 shows the result of the experiment. It can be seen that the resonance shifts to higher wavelength as the droplet increases. This effect can be interpreted as the combination of two phenomena, i.e. 1) a modification of the optical mode inside the waveguide leading to a change in the resonance of the cavity and 2) a bigger droplet forcing liquid into the slots preventing air pockets and increasing the infiltration.

To verify the first phenomenon, we have performed FDTD calculations considering different droplet sizes. A volume of water corresponds to a particular length of wet waveguide. This distance depends on the contact angle between the liquid and the substrate. Note that in our simulations, it was not possible to consider droplets as big as in the experiments, principally due to the calculation time and volume. The results are presented in Figure 6 with the scales (volume and wet distance). One can see that for very small droplets, which are not completely covering the cavity, the size of the droplet has an effect: the resonance position is shifted to higher wavelengths. However, when water completely covers the cavity this effect is considerably reduced and tends to disappear for droplets having a volume corresponding to a length of wet waveguide larger than $1 \mu \mathrm{m}$. The experiment has been done with bigger droplets. We can conclude that even if the wet distance of the waveguide affects the mode (inside the dielectric waveguide), it does not affect the resonance position.

In the next section, the second hypothesis will be verified.

\subsection{Effect of the infiltration}

The problem of the infiltration of the analyte inside the slots has been theoretically solved by performing FDTD calculations and considering different percentage of the slot 


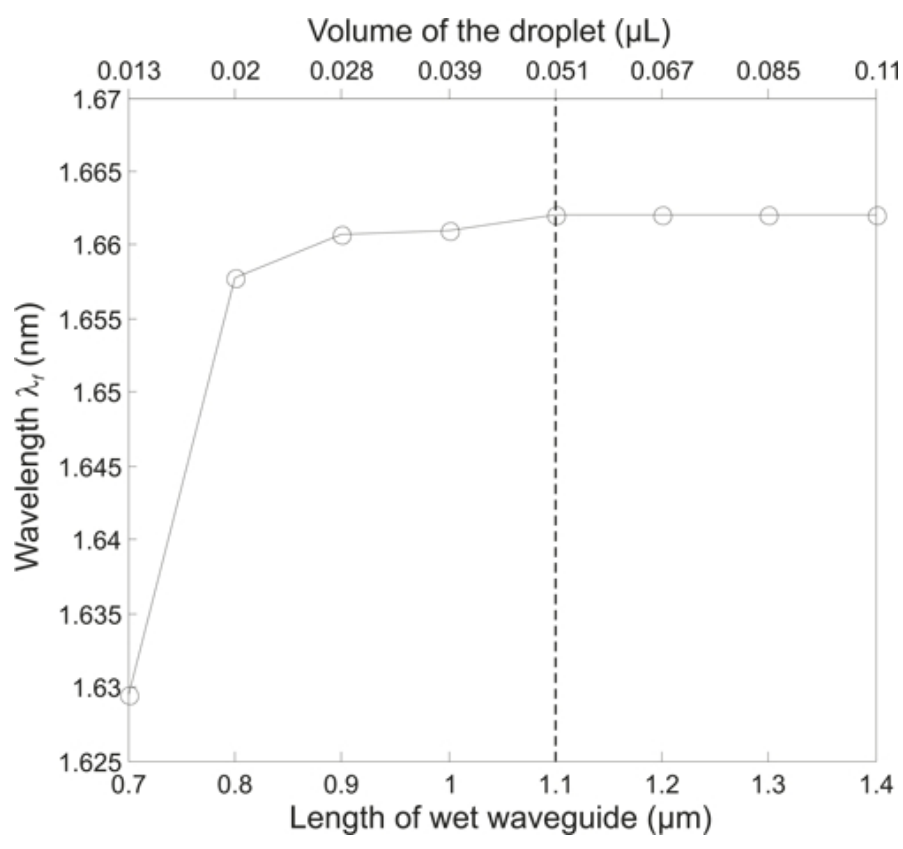

FIG. 6 Influence of the area of the waveguide covered by water on the response of the device: calculation of the resonance position.

filled with water. Due to the size of the structure, we have neglected the shape of the meniscus inside the slots. The results are shown in Figure 7.

For $0 \%, 25 \%, 50 \%, 75 \%$ and $100 \%$ of the volume of the slots filled, one can observe a perfect linear dependence between this volume and the position of the resonance, which is shifted to the higher wavelengths: the more the liquid infiltrates the cavity, the higher the resonance wavelength will be. Note that these calculation have been made for water only and we can make the same remark as for the size of the droplet and conclude that the infiltration will depend on the nature of the analyte. A shift of $100 \mathrm{~nm}$ is observed between the two extreme cases.

\subsection{Sensitivity and limit of detection}

Two important parameters have to be taken into account while characterizing a sensor. The first one is the sensitivity of the device, which is an intrinsic parameter of the structure. It will principally depends on the design, the geometrical parameters, and the overlap between the electromagnetic field and the analyte. Typically, the enhancement of the field overlap in the detection medium increases the sensitivity of the resonator based sensor. Our sensor has a measured sensitivity of $S=730 \pm 10 \mathrm{~nm} / \mathrm{RIU}$. The second parameter is the limit of detection [18], which depends mainly on the equipment used to perform the measurement. In our case, we have used as a detector an optical spectrum analyzer. Its resolution is $\sigma=50 \mathrm{pm}$. We can then achieve (theoretically) with our setup a limit of detection of $L O D=\sigma / S \simeq 10^{-5}$ RIU, which is a common value for devices like ring resonators $[19,20]$. In this paper, the smallest observed variation was $10^{-3}$ RIU.
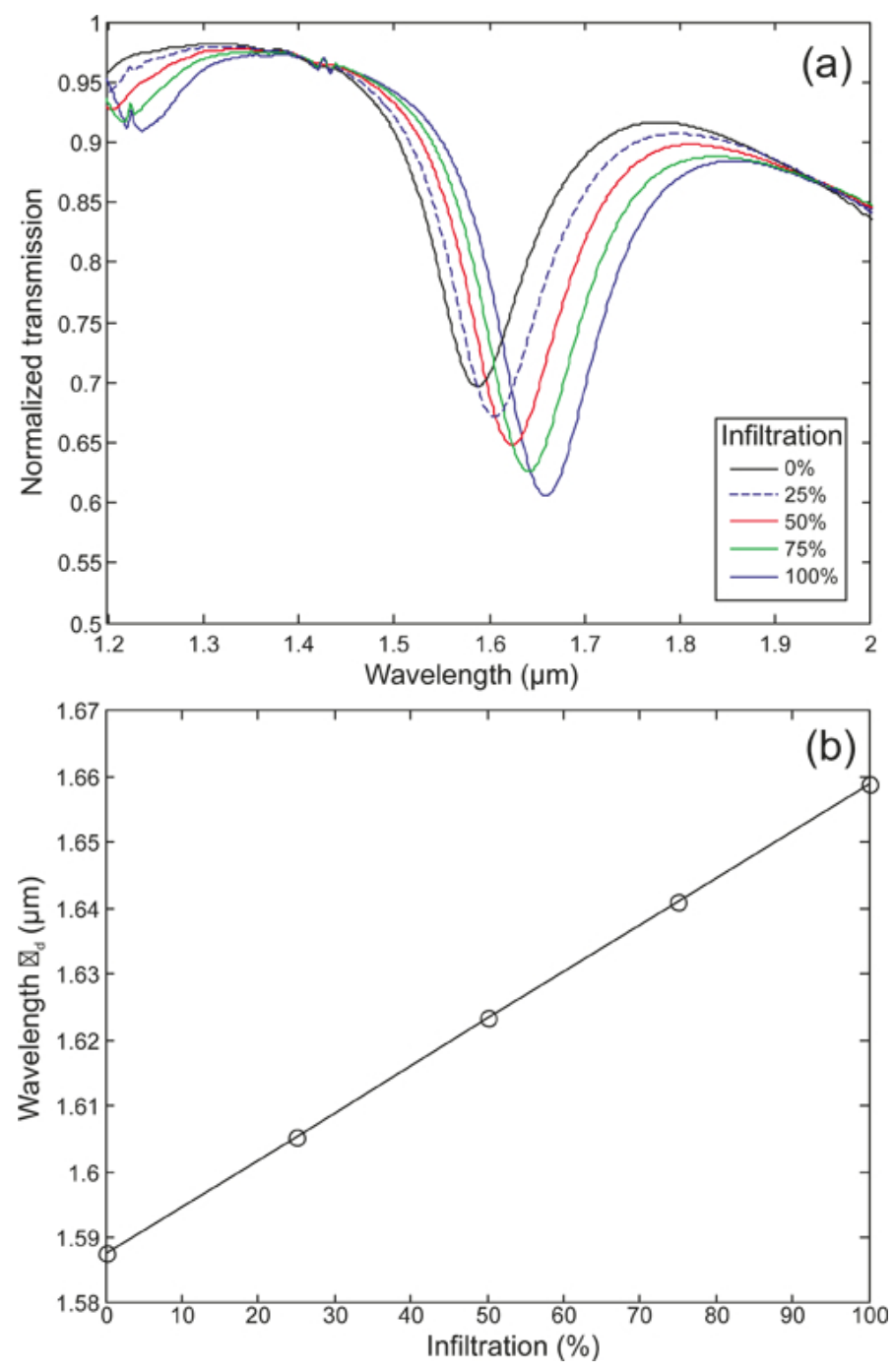

FIG. 7 Calculated response of the device in function of the infiltration of the liquid in the slots (a); Spectral position of the dip versus the percentage of infiltration (b).

\section{CONCLUSION}

In this paper we demonstrated experimentally that a slot waveguide cavity can act as a refractive index sensor for liquids over a wide range using a very small amount of analyte, which is of great interest for biosensing applications. We have established the calibration curve of the sensor and determined a sensitivity of $730 \mathrm{~nm} /$ RIU. In this study we have taken into account several parameters such as the losses, the amount of analyte or the infiltration, which play a crucial role on the sensitivity or the limit of detection of the sensor. Based on these facts, we have been able to define the optimum working condition. Moreover, we have demonstrated the potential offered by the cascaded SWC in order to increase the contrast of the measurements and thus to facilitate the resonance detection.

\section{ACKNOWLEDGEMENTS}

The authors wish to thank the Swiss National Science Foundation (SNSF) and the European Space Agency (ESA) for their financial support. Technical support was provided by the Center of Micro Nano Technology (Ecole Polytechnique Fédérale de Lausanne). 


\section{References}

[1] X. D. Fan, I. M. White, S. I. Shopoua, H. Y. Zhu, J. D. Suter, and Y. Z. Sun, "Sensitive optical biosensors for unlabeled targets: A review," Anal. Chim. Acta 620, 8-26 (2008).

[2] G. Gauglitz, "Direct optical detection in bioanalysis: an update," Anal. Bioanal. Chem. 398, 2363-2372 (2010).

[3] S. Roh, T. Chung, and B. Lee, "Overview of the Characteristics of Micro- and Nano-Structured Surface Plasmon Resonance Sensors," Sensors 11, 1565-1588 (2011).

[4] J. Homola, "Surface plasmon resonance sensors for detection of chemical and biological species," Chem. Rev. 108, 462-493 (2008).

[5] S. Herminjard, L. Sirigu, H. P. Herzig, E. Studemann, A. Crottini, J. P. Pellaux, T. Gresch, et al., "Surface Plasmon Resonance sensor showing enhanced sensitivity for $\mathrm{CO}(2)$ detection in the midinfrared range," Opt. Express 17, 293-303 (2009).

[6] T. Yoshie, L. Tang, and S.-Y. Su, "Optical Microcavity: Sensing down to Single Molecules and Atoms," Sensors 11, 1972-1991 (2011).

[7] M. Espinosa Bosch, A. J. Ruiz Sánchez, F. Sánchez Rojas, and C. Bosch 0jeda, "Recent development in optical fiber biosensors," Sensors 7, 797-859 (2007).

[8] D. F. Dorfner, T. Hüerlimann, T. Zabel, L. H. Frandsen, G. Abstreiter, and J. J. Finley, "Silicon photonic crystal nanostructures for refractive index sensing," Appl. Phys. Lett. 93, 181103 (2008).

[9] H. Jiang, and J. Sabarinathan, "Effects of Coherent Interactions on the Sensing Characteristics of Near-Infrared Cold Nanorings," J. Phys. Chem. C 114, 15243-15250 (2010).

[10] J. A. Dionne, L. A. Sweatlock, H. A. Atwater, and A. Polman, "Plasmon slot waveguides: Towards chip-scale propagation with subwavelength-scale localization," Phys. Rev. B 73, (2006).

[11] G. Veronis, and S. Fan, "Modes of subwavelength plasmonic slot waveguides," J. Lightwave Technol. 25, 2511-2521 (2007).
[12] V. R. Almeida, Q. F. Xu, C. A. Barrios, and M. Lipson, "Guiding and confining light in void nanostructure," Opt. Lett. 29, 1209-1211 (2004).

[13] Q. Tan, A. Cosentino, M. Roussey, and H. P. Herzig, "Theoretical and experimental study of a $30 \mathrm{~nm}$ metallic slot array," J. 0pt. Soc. Am. B 28, 1711-1715 (2011).

[14] A. Taflove, and S. C. Hagness, Computational Electrodynamics: The Finite-Difference Time-Domain Method (Artech House, Boston, London, 2005).

[15] A. Perentes, I. Utke, B. Dwir, M. Leutenegger, T. Lasser, P. Hoffmann, F. Baida, et al., "Fabrication of arrays of sub-wavelength nano-apertures in an optically thick gold layer on glass slides for optical studies," Nanotechnology 16, 273-277 (2005).

[16] Y. A. Vlasov, and S. J. McNab, "Losses in single-mode siliconon-insulator strip waveguides and bends," Opt. Express 12, 1622-1631 (2004).

[17] A. L. Sumner, E. J. Menke, Y. Dubowski, J. T. Newberg, R. M. Penner, J. C. Hemminger, L. M. Wingen, et al., "The nature of water on surfaces of laboratory systems and implications for heterogeneous chemistry in the troposphere," Phys. Chem. Chem. Phys. 6 , 604-613 (2004).

[18] I. M. White, and X. Fan, "On the performance quantification of resonant refractive index sensors," Opt. Express 16, 1020-1028 (2008).

[19] D.-X. Xu, M. Vachon, A. Densmore, R. Ma, S. Janz, A. Delâge, J. Lapointe, et al., "Real-time cancellation of temperature induced resonance shifts in SOl wire waveguide ring resonator label-free biosensor arrays," Opt. Express 18, 22867-22879 (2010).

[20] M. Sumetsky, R. S. Windeler, Y. Dulashko, and X. Fan, "Optical liquid ring resonator sensor," Opt. Express 15, 14376-14381 (2007). 